Research Article

\title{
Expression pattern of drought stress marker genes in soybean roots under two water deficit systems
}

Anna Cristina Neves-Borges ${ }^{1^{*}}$, Fábia Guimarães-Dias ${ }^{2 *}$, Fernanda $\mathrm{Cruz}^{2}$, Rosilene Oliveira Mesquita ${ }^{5}$, Alexandre Lima Nepomuceno ${ }^{3}$, Eduardo Romano ${ }^{4}$, Marcelo Ehlers Loureiro ${ }^{5}$, Maria de Fátima Grossi-de-Sá ${ }^{4}$ and Márcio Alves-Ferreira ${ }^{2}$

${ }^{1}$ Departamento de Botânica, Universidade Federal do Rio de Janeiro, Rio de Janeiro, RJ, Brazil.

${ }^{2}$ Departamento de Genética, Instituto de Biologia, Universidade Federal do Estado do Rio de Janeiro, Rio de Janeiro, RJ, Brazil.

${ }^{3}$ Embrapa Soja, Distrito de Warta, Londrina, PR, Brazil.

${ }^{4}$ Embrapa Recursos Genéticos e Biotecnologia, Brasília, DF, Brazil.

${ }^{5}$ Departamento de Biologia Vegetal, Universidade Federal de Viçosa, Viçosa, MG, Brazil.

\begin{abstract}
The study of tolerance mechanisms for drought stress in soybean is fundamental to the understanding and development of tolerant varieties. Using in silico analysis, four marker genes involved in the classical ABA-dependent and ABA-independent pathways of drought response were identified in the Glycine max genome in the present work. The expression profiles of the marker genes ERD1-like, GmaxRD20A-like, GmaxRD22-like and GmaxRD29B-like were investigated by qPCR in root samples of drought sensitive and tolerant soybean cultivars (BR 16 and Embrapa 48, respectively), submitted to water deficit conditions in hydroponic and pot-based systems. Among the four putative soybean homologs to Arabidopsis genes investigated herein, only GmaxRD29B-like was not regulated by water deficit stress. Distinct expression profiles and different induction levels were observed among the genes, as well as between the two drought-inducing systems. Our results showed contrasting gene expression responses for the GmaxRD20A-like and GmaxRD22-like genes. GmaxRD20A-like was highly induced by continuous drought acclimating conditions, whereas GmaxRD22-like responses decreased after abrupt water deprivation. GmaxERD1-like showed a different expression profile for the cultivars in each system. Conversely, GmaxRD20A-like and GmaxRD22-like genes exhibited similar expression levels in tolerant plants in both systems.
\end{abstract}

Key words: water deficit, drought adaptation, marker genes, Glycine max.

\section{Introduction}

For soybean (Glycine max [L.] Merrill), one of the most important agricultural commodities in the world (Clemente and Cahoon, 2009), drought is considered as one of the main causes of yield loss in different countries (Bray et al., 2000) In Brazil, the lack of rainfall in 2009 caused a drop of $4.2 \%$ in soybean crop production (Brazilian Institute of Geography and Statistics - IBGE, 2010). The losses in soybean yield in the North and West of the state of Paraná (Brazil) related to drought were $80 \%$ in 2008-2009, as compared to the average productivity of the region.

Send correspondence to Márcio Alves-Ferreira. Laboratório de Genética Molecular Vegetal, Departamento de Genética, Instituto de Biologia, Universidade Federal do Rio de Janeiro, Av. Prof. Rodolpho Paulo Rocco s/n, - Prédio do CCS, $2^{\circ}$ andar, sala A2-93, 219410-970 Rio de Janeiro, RJ, Brazil. E-mail: marcioaf@ufrj.br.

*These authors contributed equally to this work.
These losses due to drought resulted in a cumulative decline of almost 11 million tons in total production (Franchini et al., 2009). According to Manavalan et al. (2009), the drop in production is probably intensified by climate change caused by global warming and the consequent increase in frequency and extension of the water-limited regions. Therefore, comprehension of drought stress response mechanisms is expected to drive the development of drought tolerant soybean cultivars that will be crucial to maintaining soybean yield levels and countering the threat of global warming for this crop (Cutforth et al., 2007).

It has been demonstrated that plants can naturally develop drought tolerance mechanisms, allowing them to prevent or minimize the damaging effects of water deprivation. These response mechanisms involve molecular, cellular and physiological changes, triggered by a molecular signaling cascade (Bray, 1993; Seki et al., 2003; Yamaguchi-Shinozaki and Shinozaki, 2006; Manavalan et al., 
2009). This complex network of responses to drought stress may involve the abscisic acid (ABA) phytohormone, which orchestrates the production and accumulation of important molecules that trigger and amplify a signaling cascade (Mahajan and Tuteja, 2005; Adie et al., 2007; Urano et al., 2009).

The response to drought stress relies mainly on gene expression regulation, of thousands of genes (Shinozaki and Yamaguchi-Shinozaki, 2007). In Arabidopsis, this complex cascade involves the expression of transcription factors that are responsible for regulating downstream genes such as $R D 20 A, R D 22$ and $R D 29 B$ in the ABAdependent pathway, as well as ERD1 in the ABA-independent pathway (Shinozaki and Yamaguchi-Shinozaki, 2007). These four genes, $R D 20 A, R D 22, R D 29 B$ and $E R D 1$, have been widely used as water-deficit markers in Arabidopsis and other species, hence, their identification in soybean will be of importance for future research in this crop (Pellegrineschi et al., 2004; Huang et al., 2011; StolfMoreira et al., 2011).

Expression profiles of the drought-stress response genes have been investigated under water-deficit stress conditions established in the hydroponic systems (HSys) (Fujita et al., 2004; Ito et al., 2006; Stolf-Moreira et al., 2010a,b, 2011). Notwithstanding, some authors prefer to assess drought responses in pot-based systems (PSys) because they better reproduce field conditions (Casagrande $e t$ al., 2001; Qin et al., 2007; Tran et al., 2009). In the PSys condition, drought promotes a slower water deprivation process, allowing the plant to adapt to the water deficit. Conversely, in the HSys condition, the water deficit occurs abruptly by removing the plant from the nutritient solution or by the addition of osmolytes to the solution (Cowan, 1965). The instantaneous water deficit in HSys causes severe consequences to the soybean plants, impeding a continuous acclimation process (Munns et al., 2010). Therefore, the water-deficit stress responses to the PSys and HSys conditions may differ significantly, triggering expression of distinct sets of genes (Martins et al., 2008; Munns et al., 2010).

By means of an in silico approach we identified herein the soybean (G. max) homologs of the Arabidopsis $E R D 1, R D 20 A, R D 22$ and $R D 29 B$ genes. These genes are classical markers for the ABA-dependent and ABA-independent pathways of response to drought (Pellegrineschi et al., 2004; Huang et al., 2011). The expression profile for each soybean gene under water deficit stress condition and contrasting the PSys and HSys conditions was assayed by qPCR analysis. The identification and molecular characterization of these drought marker genes (DMGs) under distinct water-deficit stress systems are important not only to reveal differences between the two experimental procedures, but also to provide bona fide marker genes for those interested in studying drought stress in soybean.

\section{Material and Methods}

\section{Identification of DMGs in soybean response to drought}

For the identification of DMGs in the response of soybean to drought we employed a search strategy illustrated in Figure S1 (Supplementary Material). DMGs involved in the response to drought in Arabidopsis were identified based on published data (Bray, 2002; Kang et al., 2002; Fujita et al., 2005; Shinozaki and YamaguchiShinozaki, 2007) and searches using web tools of the Arabidopsis Information Resource (TAIR). The protein sequences of Arabidopsis genes (ERD1, RD20A, RD22 and $R D 29 B$ ) and their putative paralogs were used to search all possible homologs in the G. max and Oryza sativa genomes by means of the BLASTP tool. Those meeting the criterion of an E-value $\leq 10^{-18}$ in the Phytozome and TAIR sites were considered for further investigation. For constructing dendrogram we first performed a multiple alignment of the amino acid sequences for each selected gene using ClustalW2 software (Larkin et al., 2007). These data were then used to build dendrograms using MEGA version 4.0 (Molecular Evolutionary Genetics Analysis) program (Tamura et al., 2007) with the Neighbor-Joining clustering method (Crandall et al., 2008) and data derived from a p-distance matrix (Crandall et al., 2008) in a Poisson model and using the complete deletion option. Tree reconstruction was performed using the Interior Branch Test Phylogeny approach and bootstrapping (1,000 replications) (Sitnikova et al., 1995). For rooting the trees, O. sativa sequences were used as the outgroup.

\section{In silico expression analysis}

In order to investigate the pattern of induction/repression of the Arabidopsis, ERD1, RD20A, RD22 and RD29B genes, the expression data of the Arabidopsis genes during the response to different water privation conditions or $\mathrm{ABA}$ stimulus were retrieved from the Genevestigator database (Hruz et al., 2008). Data were presented as absolute expression values or fold change compared with that of the control samples by integrating expression data from thousands of transcriptomic experiments present in the Genevestigator database.

\section{Plant material and drought assays}

We used the soybean (G. max) cultivars, BR 16 and Embrapa 48, these being sensitive and tolerant to drought, respectively (Casagrande et al., 2001; Texeira et al., 2008). Drought assays were performed in two different water deficit treatments, a pot-based (PSys) and a hydroponic system (HSys) (Martins et al., 2008; Kulcheski et al., 2010).

Plants grown in the PSys condition were maintained at a controlled temperature $\left(30^{\circ} \mathrm{C} \pm 5^{\circ} \mathrm{C}\right), 60 \% \pm 20 \%$ relative humidity and natural photoperiod. Seeds from both genotypes were germinated in washed sand. After approxi- 
mately 10 days, seedlings of each genotype were transplanted to pots containing bovine fertilizer. Plants at the V4 developmental stage (fourth trifoliate leaf fully expanded) (Fehr and Caviness, 1977) were submitted to irrigation (control) or water deficit conditions, by suspension of irrigation for 7-10 days, until reaching a water potential of $-1.5 \mathrm{MPa} \pm 0.2 \mathrm{MPa}$ and $-3.0 \mathrm{MPa} \pm 0.2 \mathrm{MPa}$ (moderate and severe level stress, respectively). The water potential $\left(\Psi_{\mathrm{w}}\right)$ of each plant was measured at predawn (between 05:00 and 06:00) in the fourth or fifth leaf from the apex using a Scholander-type pressure chamber. Roots were removed from the pot and immediately rinsed with water for $1 \mathrm{~min}$ with gentle agitation to remove adhering sand. Biological contaminants were removed by immersion in SDS solution $(2 \%)$ for $1 \mathrm{~min}$ followed by a gentle wash in ultrapure water for $1 \mathrm{~min}$. Finally, the root samples were immediately frozen in liquid nitrogen and stored at $-80^{\circ} \mathrm{C}$ for RNA extraction. Two biological replicates for each condition were collected for gene expression studies.

In the HSys condition, the seeds of both cultivars were placed on moist filter paper and pre-germinated in the dark at $25{ }^{\circ} \mathrm{C} \pm 1{ }^{\circ} \mathrm{C}$ and $65 \% \pm 5 \%$ relative humidity. Seedling were transferred to polystyrene supports and their roots maintained completely immersed in a nutrient solution $(\mathrm{pH}$ balanced at 6.6 and aerated) (Kulcheski et al., 2010), under natural photoperiod daylight (photosynthetic photon flux density $\left(\right.$ PPFD) $=1.5 \times 10^{3} \mu$ moles $\mathrm{m}^{-2} \mathrm{~s}^{-1}$, equivalent to $8.93 \times 10^{4}$ lux) and a $12 \mathrm{~h}$ daylength, at $25^{\circ} \mathrm{C} \pm 2{ }^{\circ} \mathrm{C}$ and $60 \% \pm 5 \%$ relative humidity. After 2 weeks, the seedlings at the V4 developmental stage of both genotypes were removed from the HSys condition and kept in the dark without nutrient solution or water for different water deprivation periods: $0 \mathrm{~min}$ (T0, control), $50 \mathrm{~min}$ (T50), $100 \mathrm{~min}$ (T100) and 150 min (T150) of stress. To verify the water deficit, photosynthetic rate, stomatal conductance, intercellular $\mathrm{CO}_{2}$ concentration, transpiration rate and leaf temperature were evaluated using a LI-6400 Portable Photosynthesis System (LiCor, Inc.). Measurements were taken on the fully expanded middle leaflet of the basal second leaf node under a photon flux density of $1,000 \mu \mathrm{mol} \mathrm{m} \mathrm{m}^{-2} \mathrm{~s}^{-1}$. For details, see Figure 2 of Rodrigues et al. (2012). Two biological replicates for each condition were used in the expres- sion studies. The root samples corresponding to a pool composed of 10 plants from each treatment were immediately frozen in liquid nitrogen, followed by storage at $-80{ }^{\circ} \mathrm{C}$ for posterior RNA extraction.

\section{Total RNA isolation}

Root samples from the PSys condition were processed for RNA extraction using the Plant RNAeasy kit (Qiagen) following the manufacturer's protocol. RNA extraction of the root samples from the hydroponic experiments was done with Trizol Reagent (Invitrogen). In both cases, the RNA samples were treated with RNAse-free DNAse I (BioLabs) to eliminate any DNA contamination. RNA integrity was checked by electrophoresis on a $1 \%$ agarose gel, and RNA concentration and purity were determined using a NanoDrop spectrophotometer ND-1000 (Thermo Scientific).

\section{Real-time quantitative polymerase chain reaction (qPCR)}

The expression of DMGs in both soybean cultivars submitted to different drought conditions was evaluated by qPCR analysis. Primers pairs of $20 \mathrm{bp}$ and Tm of $60^{\circ} \mathrm{C} \pm$ $1{ }^{\circ} \mathrm{C}$ designed with Primer 3 plus software (Untergasser et al., 2007) were used to amplify a region of 80-200 bp of the respective target gene. For normalization of target gene expression, ACT11 (cytoskeletal structural protein) and $F B O X$ (F-Box protein family) reference genes (RGs) were used (Kulcheski et al., 2010). The stability of expression of each of these RGs under the experimental conditions was checked by means of the NormFinder program (Andersen et al., 2004) (data not shown). All primer sequences and amplicon lengths are listed in Table 1.

qPCR assays were carried out in a Realplex 4 Eppendorf Mastercycler Epgradient (Eppendorf) sequence detection system using a Power SYBR ${ }^{\circledR}$ Green RNA-to-Ct One-Step Kit (Applied Biosystems) following the manufacturer's protocol. For each sample, $25 \mathrm{ng}$ of RNA was used in the reaction mixtures in a final volume of $20 \mu \mathrm{L}$. For each primer combination, all samples were evaluated in technical triplicates and including a no-template control. Reaction mixtures were incubated for $30 \mathrm{~min}$ at $48^{\circ} \mathrm{C}$, followed by $10 \mathrm{~min}$ at $95{ }^{\circ} \mathrm{C}$ and 40 amplification cycles of

Table 1 - Primer sequences used in the qPCR protocols and amplicon lengths.

\begin{tabular}{lcll}
\hline Gene model & Soybean gene & Forward primer sequence [5'-3'] & Reverse primer sequence [5'-3'] \\
\hline RD20A & Glyma03g41030 (GmaxRD20A-like) & GTGGCACATGACTGAAGGAA & ATCTTTCCAGCAGCACCTCT \\
RD22 & Glyma14g20450 (GmaxRD22-like) & AATGCCGAAAGCCATTACAG & GCTTTGTTTTCCCTGCGTTA \\
RD29B & Glyma16g31330 (GmaxRD29B-like) & AGCTGACAAAGCCATCACTG & CTCTGTCAGGGACTGAGCAA \\
ERD1 & Glyma04g38050 (GmaxERD1-like) & CGTCCAGAATTGCTCAACAG & TGGGGTTATAGCCTTGTTGG \\
ACT11 & Glyma18g52780 & CGGTGGTTCTATCTTGGCATC & GTCTTTCGCTTCAATAACCCTA \\
FBOX & Glyma12g05510 & CTAATGGCAATTGCAGCTCTC & AGATAGGGAAATGGTGCAGGT \\
\hline
\end{tabular}


$15 \mathrm{~s}$ at $95^{\circ} \mathrm{C}$ and $1 \mathrm{~min}$ at $60^{\circ} \mathrm{C}$ (fluorescence measurement step). At the end of 40 cycles, a melting-curve analysis was run $\left(15 \mathrm{~s}\right.$ at $95{ }^{\circ} \mathrm{C}, 15 \mathrm{~s}$ at $60{ }^{\circ} \mathrm{C}$ - fluorescence measurement step, and $15 \mathrm{~s}$ at $95^{\circ} \mathrm{C}$ ). Melting curve and $1 \%$ gel electrophoresis analysis of the amplification products were employed to confirm the presence of only a single amplified product of expected size. Primer set efficiencies were estimated for each experimental set by using the Miner software (Zhao and Fernald, 2005) through a nonlinear regression algorithm without the need for a standard curve, and the values were used in all subsequent analyses. In addition, the values of the threshold cycle (quantification cycle value $-\mathrm{Cq}$ ) were converted by the program QBASE v1.3.5 (Hellemans et al., 2007), into normalized relative quantities (NRQ).

\section{Promoter analysis}

The promoter sequences of the DMG (1000 bp length upstream from the start codon) were obtained from the genome browser tool in the Phytozome database. Cis-regulatory elements responsive to drought stress, salinity stress, osmotic stress and ABA (Table S1) were identified through a web tool in the database of Plant Cis Program-acting Regulatory DNA Elements - PLACE (Higo et al., 1999) and also from published data (Busk and Pages, 1998; Li and Chen, 1999; Choi et al., 2000; Simpson et al., 2003; Nakashima et al., 2006; Lenka et al., 2009; Mochida et al., 2009; Umezawa et al., 2010).

The frequency of the respective cis-regulatory elements in the promoter region of each gene of interest was compared to the expected frequency in the genes of the $G$. $\max$ genome. The statistical analysis of cis-elements of the gene of interest promoters was performed by the POBO web tool (Kankainen and Holm, 2004).

\section{Results}

Identification and in silico characterization of drought marker genes from ABA-dependent and ABA-independent pathways involved in the drought-stress response in soybean

In order to identify and characterize Drought Marker Gene (DMG) homologs for ERD1, RD20A, RD22 and $R D 29 B$ in soybean we initially used an in silico approach, followed by qPCR validation. We also evaluated the promoter region of these genes for the presence and frequency of cis-elements related to drought stress. The diagram of the search strategy employed is illustrated in Figure S1 (Supplementary Material).

To assess the expression pattern of the ERDI, $R D 20 A, R D 22$ and $R D 29 B$ genes under different waterdeficit stress conditions and ABA stimulus in Arabidopsis, we used the Genevestigator web tool (Hruz et al., 2008). The digital expression analyses confirmed previous results showing that $R D 20 A, R D 22$ and $R D 29 B$ are induced by drought stress and ABA, whereas ERD1 is induced predominantly by drought stress (Figure $\mathrm{S} 2$ ).

The Arabidopsis gene models, as well as their respective amino acid sequences and functions, were crucial for the search for putative homologs in the soybean genome. The putative homologs for each Arabidopsis gene in the soybean genome were identified through a BLASTP search in the Phytozome database combined with a Neighborjoining analysis. For each Arabidopsis gene under consideration we identified the putative homologs in the G. $\max$ and $O$. sativa genomes. The threshold $e$-value used for the identification of the putative homologs and their use in a dendrogram analysis was determined according to the size of the gene families evaluated. For instance, ERD1 belongs to a large gene family, indicating the use of an e-value threshold of $\leq 10^{-50}$. In contrast, for gene families with only few members, such as the RD20A or RD22 protein families, the e-value threshold was set at $\leq 10^{-30}$. Finally, the $R D 29 B$ gene presents only a few putative homologs with very low similarity, hence an e-value threshold of $\leq 10^{-18}$ was indicated.

The dendrogram analysis was performed on the selected protein sequences to uncover putative soybean homologs for the Arabidopsis DMGs (Figure 1). When the dendrogram analysis could not pinpoint a single putative homolog, the top e-value obtained in the BLASTP analysis was consider as selection criteria. This strategy allowed us to identify the four putative soybean homolog genes, GmaxERD1-like (Glyma04g38050), GmaxRD20A-like (Glyma03g41030), GmaxRD22-like (Glyma14g20450) and GmaxRD29B-like (Glyma16g31330), for the Arabidopsis DMGs ERD1 (AT5G51070), RD20A (AT2G33380), RD22 (AT5G25610) and $R D 29 B$ (AT5G52300), respectively (Figure 1). As previously mentioned, the $R D 20 A, R D 22$ and $R D 29 B$ genes belong to the ABA-dependent pathways, while the $E R D 1$ gene belongs to the ABA-independent pathways of drought stress response.

\section{Expression profile analysis of putative soybean DMGs by real-time quantitative polymerase chain reaction (qPCR)}

The expression pattern of each soybean DMG involved in the drought response was investigated by qPCR. The analysis was performed on RNA samples from roots of two soybean cultivars that presented contrasting responses to drought, the cultivar BR 16 being highly sensitive and the cultivar Embrapa 48 moderately tolerant to drought (Casagrande et al., 2001; Texeira et al., 2008). These plants had been submitted to different water deficit conditions (PSys and HSys) and also several stress levels.

Among the four DMGs identified by our analysis, GmaxRD29B-like was not induced under any of the test conditions (data not shown), whereas Gmaxerdl-like, GmaxRD20A-like and GmaxRD22-like were induced in both cultivars in the two cultivation conditions, PSys and 


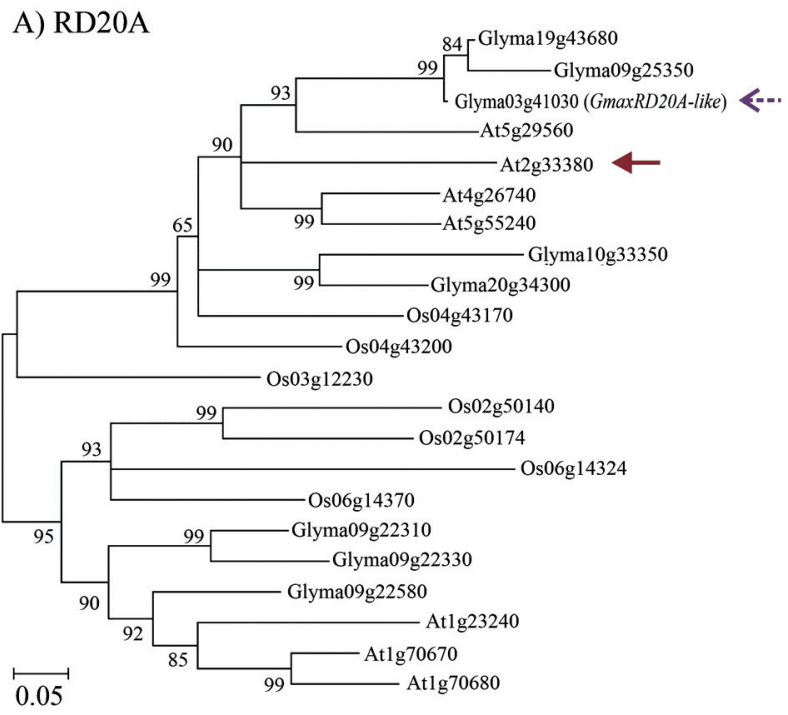

B) RD22

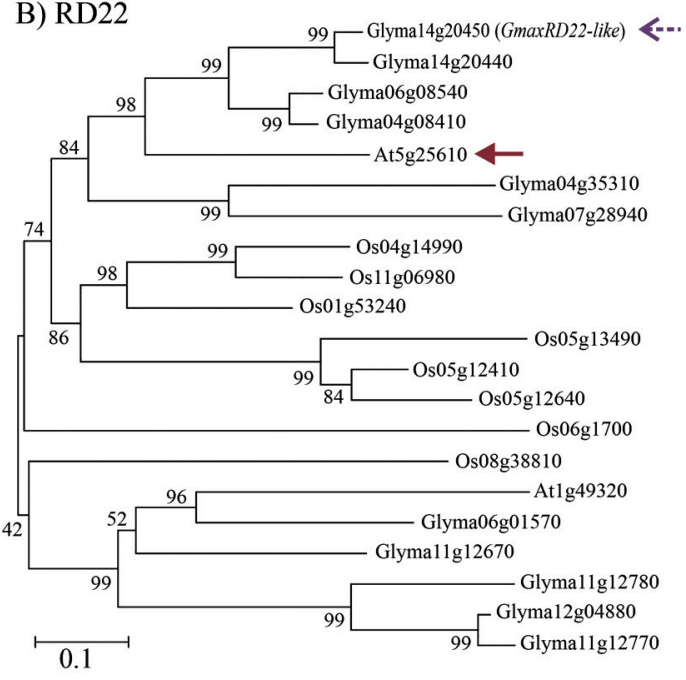

C) RD29B

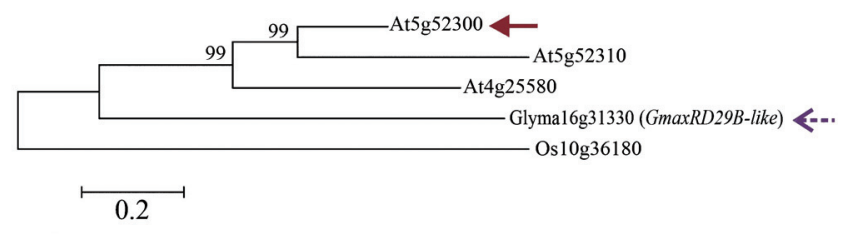

D) ERD1

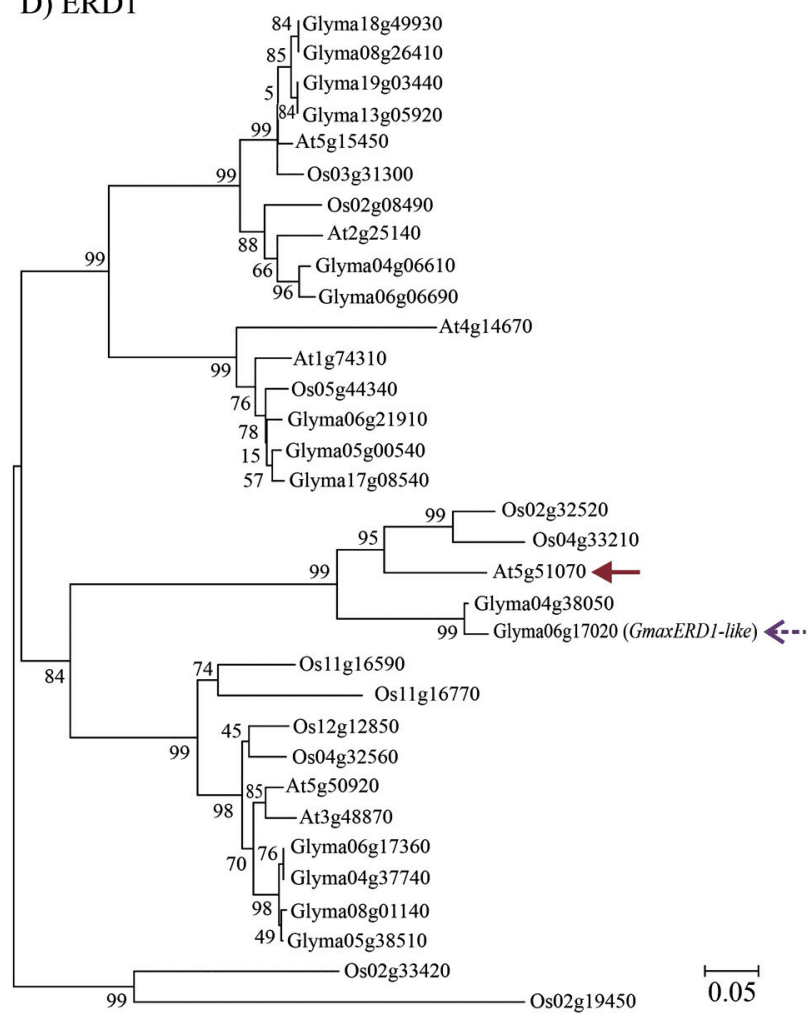

Figure 1 - Dendrograms of the Arabidopsis thaliana, Glycine max and Oryza sativa key genes responsive to drought, based on the amino acid sequences. The multiple alignment was made using ClustalW2 and the dendrograms were built using MEGA4.0 software, with the Neighbor-Joining and pair-wise deletion options as a consensus of 1,000 bootstrap replicates. $O$. sativa was used as outgroup. The solid brown arrows indicate the Arabidopsis reference key genes and the dotted purple arrows indicate the respective soybean homologs selected for qPCR validation of expression. A) Dendrogram of the RD20A family of A. thaliana, G. max and O. sativa; B) Dendrogram of the RD22 family of A. thaliana, G. max and O. sativa; C) Dendrogram of the RD29B family of A. thaliana, G. max and O. sativa; D) Dendrogram of the ERD1 family of A. thaliana, G. max and O. sativa.

HSys. Nevertheless, these genes showed distinct expression dynamics and induction levels depending on drought conditions assessed.

The GmaxRD20A-like gene was induced progressively in both soybean cultivars under PSys. It reached the highest expression level in the sensitive cultivar under the most severe stress condition ( $\left.\Psi_{\mathrm{w}}-3.0 \mathrm{MPa}\right)$ in the PSys (Figure 2A). In contrast, the gene expression level under HSys was very low for all tested conditions (Figure 2A).

The GmaxRD22-like gene showed quite similar expression levels and dynamics for the two cultivars in the PSys condition, with high expression restricted to the severe water deficit condition, $\Psi \mathrm{w}-3.0 \mathrm{MPa}$ (Figure 2B). In HSys, GmaxRD22-like presented very low expression lev- els in both tolerant and sensitive cultivars. Interestingly, the two genes that belong to the ABA-dependent pathway, GmaxRD22-like and GmaxRD20A-like showed similar expression profiles in the test conditions.

In contrast to the other genes evaluated in this work, the GmaxERD1-like, which belongs to the ABA-independent pathway, was highly expressed in the HSys condition. It is worthy of note that this gene was about five times more expressed in the sensitive cultivar than in the tolerant one under the most severe stress condition, T150 (Figure 2C).

\section{Promoter analysis}

We employed the POBO program (Kankainen and Holm, 2004) to evaluate the enrichment of 17 cis-elements 
A) GmaxRD20A-like

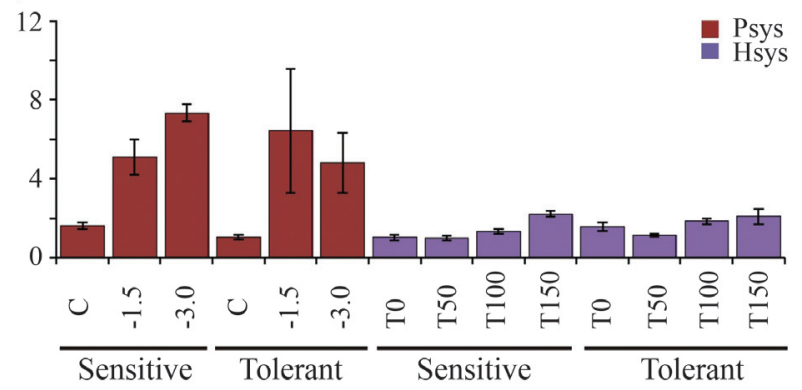

B) GmaxRD22A-like

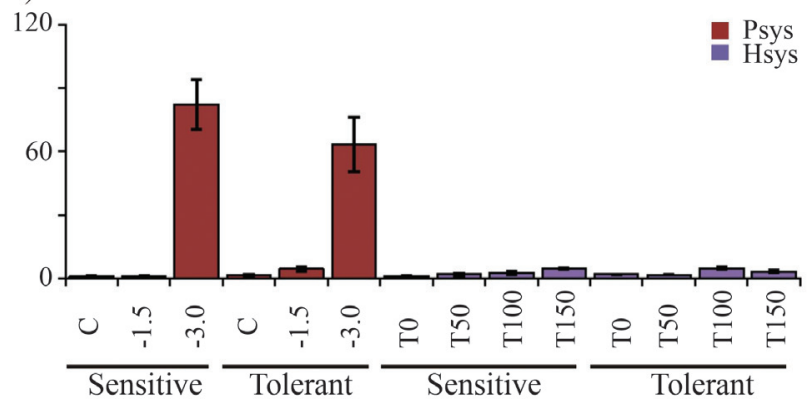

C) GmaxERD1-like

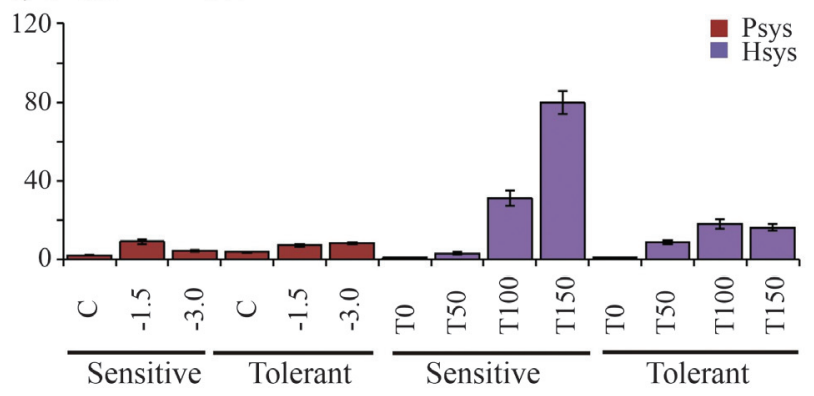

Figure 2 - Expression profile analyses for the soybean genes GmaxRD20A-like (A), GmaxRD22-like (B) and GmaxERD1-like (C) in the roots of sensitive and tolerant cultivars during water deficit stress in pot-based (PSys) and hydroponic (HSys) systems. Marker genes responsive to soybean genotypes are differentially regulated in roots, during water deficit stress conditions established in PSys (C - control no stress; $\Psi \mathrm{w}$ $-1.5 \mathrm{MPa}$ and $\Psi_{\mathrm{W}}-3.0 \mathrm{MPa}$ ) or in HSys (T0, T50, T100; and T150 min after water privation, respectively). The relative expression values, represented on the Y-axis, were obtained by qPCR experiments and calculated using the $2^{\mathrm{Ct}}$ method with $A C T$ and $F B O X$ as endogenous control genes for normalization. The qPCR assay for each gene was performed in triplicate for each of the two independent biological replicates used in the validation. Means and Standard errors of three technical replications are shown. The brown and purple bars indicate the pot-based and hydroponic systems, respectively.

related to drought, $\mathrm{ABA}$, or osmotic stress, such as ABRE, DRE, MYB, MYC or NAC cis-elements (Table S1), that we had found previously in the PLACE data bank (Higo et al., 1999). The statistical significance of their enrichment in the promoters of GmaxRD20A-like, GmaxRD22-like and GmaxERD1-like was also evaluated. GmaxRD29B-like was excluded from this analysis because it was not responsive to the drought conditions evaluated in this work. To do so, we compared the frequency of the 17 motifs in a group comprising these genes and in a background set (comprising all promoters regions of the G. $\max$ genome - BG model). This analysis revealed that three cis-elements (ACGT, WAACCA and CANNTG) are significantly enriched in the promoter region of the three genes analyzed when compared to the background set, while the ACCGAC motif is significantly enriched in the promoter region of the GmaxRD20A-like and GmaxRD22-like genes, also compared to the background set (Table S2). The most striking result was found for the ACGT motif, an element functionally that is important in a variety of promoters responding to ABA, among other stimuli (Guiltinan et al., 1990). The analysis in POBO indicated that the ACGT motif is present up to 16 times in the promoter regions of the DMG genes, with an average of 5.33 compared to an average of 3.02 for the genome background (Table S2).

\section{Discussion}

We herein identified and characterized the G. max putative homologs of Arabidopsis DMGs. The putative soybean homologs of the Arabidopsis genes RD20A, RD22 and $E R D 1$ were induced during drought stress in the two experimental systems tested: the PSys and the HSys conditions. These four genes have been employed as markers of water deficit in several works in Arabidopsis and other species (Pellegrineschi et al., 2004; Huang et al., 2011; StolfMoreira et al., 2011).

In order to identify the soybean homologs for each Arabidopsis gene previously characterized we performed a dendrogram analysis for each gene family, using the genome information for Arabidopsis, rice and soybean. We could not find a bona fide soybean homolog for the Arabidopsis RD29B gene. Based on the dendrogram analysis we selected the soybean genes that showed the highest degree of similarity for each Arabidopsis DMG (Figure 1), aiming to uncover functional similarities. The selected genes had their expression evaluated by qPCR assays in two cultivars grown under two different water-deficit stress systems.

In PSys, the drought conditions should occur in a mode similar to field conditions, triggering genes involved in a continuous acclimation process. Since PSys can entail problems of heterogeneity and inconstant water potential, among other interferences, this has led researchers to use also use HSys in water-deficit stress experiments (Martins et al., 2008; Munns et al., 2010). HSys allows for quick drought induction and straightforward sample collection (Martins et al., 2008; Munns et al., 2010). However, in HSys, the water deficit stress is promoted suddenly by removing the plant from the nutrient solution, and it completely differs from field conditions, causing shock and injuries that do not allow a continuous acclimation process. After 100 min in HSys without the nutrient solution, the water deficit is so severe that it leads to wilting in soybean plants (Martins et al., 2008). Since there is no consensus 
about the best system to promote water deficit, we decided to investigate the expression profiles of genes responsive to drought in both water-deficit stress induction systems. Therefore, besides providing the identification of soybean DMGs, our work also revealed discrepancies in gene expression patterns between the two systems.

The expression profiles of the putative soybean homologs GmaxRD20A-like, GmaxRD22-like and GmaxERD1-like were notably different in HSys when compared to PSys (Figure 2). GmaxRD20A-like and GmaxRD22-like putative homologs of Arabidopsis genes from the ABA-dependent pathway, were predominantly expressed in PSys, whereas GmaxERD1-like, a putative homolog of an Arabidopsis gene from the ABA-independent pathway, was mainly expressed in HSys (Figure 2C). The expression levels and profiles also varied among genes. For instance, GmaxRD22-like was highly expressed in both cultivars in PSys, and its expression levels in severe stress $\left(\Psi_{\mathrm{W}}-3.0 \mathrm{MPa}\right)$ were nine to eleven times higher than in moderate stress $\left(\Psi_{\mathrm{W}}-1.5 \mathrm{MPa}\right)$. In contrast, GmaxRD20A-like showed lower expression levels and only a minor difference in gene expression between severe and moderate stress. Considerable differences in DMG expression between the sensitive and tolerant soybean cultivars were exclusively found for GmaxERD1-like in plants grown under HSys. The difference was limited to the expression levels, but this may have biological significance. These results suggest that drought stress levels and pathway activation may vary considerably between the two systems investigated in this work. Martins et al. (2008) also observed differences in the expression profiles of Axi 1, PITP and $b H L H$ soybean genes between the two systems and reported significant differences among time points in stress recognition and the subsequent adaptive response between mild or more severe stress levels. GmaxERD1-like showed expression levels of about four times higher in the sensitive cultivar in the T150 condition of HSys (Figure $2 \mathrm{C}$ ), suggesting that the sensitive cultivar may have a stronger response to water deficit stress at the molecular level due to the lack of traits that are important for plant adaptation to drought. Another explanation could be the occurrence of damage-related responses that may not have been induced in the tolerant cultivar under the same stress level. GmaxRD20A-like and GmaxRD22-like did not show such responses, which may indicate that GmaxERD1-like belongs to an alternative response pathway. In fact, GmaxERD1-like should be part of the ABA-dependent and GmaxRD20A-like and GmaxRD22-like of the ABA-independent pathway. However, more genes from each pathway are needed to confirm this hypothesis. Stolf-Moreira et al. (2011) identified other putative Arabidopsis ERD1 homolog genes in the G.max genome, named GmERD1, which showed low expression in the tolerant cultivar in all drought conditions tested in the HSys condition. GmERD1 was not included in our analysis because it has a very low similarity to Arabidopsis ERD1 (data not shown).

GmaxRD22-like gene expression was highly induced in plants grown under severe stress in PSys. When compared to other genes evaluated herein, GmaxRD22-like could be ranked as the best DMG for PSys. However, no significant differences were observed between sensitive and tolerant cultivars (Figure 2B). Despite the fact that a gradual increase in expression of GmaxRD22-like was observed in HSys, the discrepancy seen in expression levels highlights the differences of the two experimental procedures employed in this work.

GmaxRD20A-like showed a similar expression profile in the sensitive and tolerant cultivars. However, GmaxRD20A-like was more expressed in the tolerant cultivar under severe stress ( $\left.\Psi_{\mathrm{w}}-3.0 \mathrm{MPa}\right)$ in PSys (Figure $2 \mathrm{~A}$ ). In Arabidopsis, the RD20A gene is regulated by the AREB1/ABF2 transcription factor, which is also involved in the regulation of the RD29B gene (Fujita et al., 2005; Shinozaki and Yamaguchi-Shinozaki, 2007). Even after exhaustive searches in the soybean genome, the most similar gene we found to Arabidopsis RD29B, Glymal6g31330, showed low similarity (e-value $10^{-18}$ ). Furthermore, this putative $R D 29 B$ soybean homolog is not regulated by the water deficit stress according to our experiments (data not shown). These results indicate that $R D 29 B$ homologs are absent in the soybean genome. Recently, it was shown that Arabidopsis RD29B is unlikely to perform any protective effects during drought stress (Msanne et al., 2011). Therefore, the lack of $R D 29 B$ homologs in the soybean genome should not interfere in the tolerance of this species to drought.

The higher frequency of the cis-elements ACGT, ACCGAC, WAACCA and CANNTG in the promoter regions of DMGs suggests that these elements may be important in expression activity during water deficit. In addition, this result also indicates that these genes probably share cis-regulatory elements related to drought stress responses. These motifs are important molecular keys involved in the transcriptional regulation of a dynamic network of gene activities (Simpson et al., 2003; Li et al., 2006; Lenka et al., 2009; Mochida et al., 2009; Umezawa et al., 2010). Additionally, these motifs have a well-documented role in regulating the expression of tolerance in response during drought stress (Busk and Pages, 1998; Simpson et al., 2003; Nakashima et al., 2006).

The soybean DMGs have in their promoter region the WAACCA and CANNTG motifs, which are MYB and MYC recognition sites, respectively. The MYC transcription factor, $\mathrm{MYC} 2$, and a MYB transcription factor, MYB2, have been shown to bind to these motifs in the Arabidopsis RD22 promoter and cooperatively activate the RD22 gene (Abe et al., 1997). Moreover, analysis of the Arabidopsis ERD1 promoter, a gene that participates in ABA-independent pathways, showed that a MYC recogni- 
tion motif is necessary for the induction of the ERD1 gene by dehydration stress (Simpson et al., 2003). Thus, the promoters of the DMGs investigated in this work share some cis-elements with their Arabidopsis homologs.

In conclusion, we identified three genes as homologs of traditional Arabidopsis DMGs, which were induced by water deficit in soybean. The GmaxRD20A-like and GmaxRD22-like genes, homologs of Arabidopsis genes of the ABA-independent pathway, are highly induced by water deficit in the PSys condition, whereas GmaxERD1-like, a homolog of an Arabidopsis gene of the ABA-dependent pathway, was highly induced by water deficit in HSys. Hence, these three genes can be very useful as stress markers for studying water deficit in soybean. Moreover, the differences in gene expression in the two systems revealed by our work emphasize that both systems need to be employed to get a bona fide picture of the pathways activated by water deficit in soybean. Finally, the differences in gene expression of the GmaxERD1-like in the HSys condition between sensitive and tolerant cultivars are robust and may provide a means to better understand the drought tolerance phenotype in soybean cultivars.

\section{Acknowledgments}

We thank Camila Maistro Patreze and Sarah Muniz Nardeli for comments on a previous version manuscript and Durvalina Felix for technical support. This work was part of Guimarães-Dias' $\mathrm{PhD}$ research in Genetics, at the Department of Genetics of the Universidade Federal do Rio de Janeiro (UFRJ), and was supported by Conselho Nacional de Desenvolvimento Científico e Tecnológico (CNPq; M. Alves-Ferreira: \# 306025/2010-8) and Fundação de Amparo à Pesquisa do Rio de Janeiro (FAPERJ).

\section{References}

Abe H, Yamaguchi-Shinozaki K, Urao T, Iwasaki T, Hosokawa D and Shinozaki K (1997) Role of Arabidopsis MYC and MYB homologs in drought- and abscisic acid-regulated gene expression. Plant Cell 9:1859-1868.

Adie BAT, Pérez-Pérez J, Pérez-Pérez MM, Godoy M, SánchezSerrano J-J, Schmelz EA and Solano R (2007) ABA Is an essential signal for plant resistance to pathogens affecting JA biosynthesis and the activation of defenses in Arabidopsis. Plant Cell 19:1665-1681.

Andersen CL, Jensen JL and Ørntoft TF (2004) Normalization of real-time quantitative reverse transcription-PCR data: A model-based variance estimation approach to identify genes suited for normalization, applied to bladder and colon cancer data sets. Cancer Res 64:5245-5250.

Bray EA (1993) Molecular responses to water deficit. Plant Physiol 103:1035-1040.

Bray EA (2002) Classification of genes differentially expressed during water-deficit stress in Arabidopsis thaliana: An analysis using microarray and differential expression data. Ann Bot 89:803-811.
Bray EA, Bailey-Serres J and Weretilnyk E (2000) Responses to abiotic stresses. In: Buchanan BB, Gruissem W and Jones RL (eds) Biochemistry and Molecular Biology of Plants. American Society of Plant Physiologists, Rockville, pp 1158-1203.

Busk PK and Pages M (1998) Regulation of abscisic acid-induced transcription. Plant Mol Biol 37:425-435.

Casagrande EC, Farias JRB, Neumaier N, Oya T, Pedroso J, Martins PK, Breton MC and Nepomuceno AL (2001) Expressão gênica diferencial durante déficit hídrico em soja. Rev Bras Fisiol Veg 13:168-184.

Choi H-I, Hong J-H, Ha J-o, Kang J-Y and Kim SY (2000) ABFs, a family of ABA-responsive element binding factors. J Biol Chem 275:1723-1730.

Clemente TE and Cahoon EB (2009) Soybean oil: Genetic approaches for modification of functionality and total content. Plant Physiol 151:1030-1040.

Cowan IR (1965) Transport of water in the soil-plant-atmosphere system. J Appl Ecol 2:221-239.

Crandall K, Lagergren J, Simonsen M, Mailund T and Pedersen C (2008) Rapid neighbour-joining. In: Crandall K and Lagergren J (eds) Algorithms in Bioinformatics, vol. 5251. Springer Berlin, Heidelberg, pp 113-122.

Cutforth HW, McGinn SM, McPhee KE and Miller PR (2007) Adaptation of pulse crops to the changing climate of the northern Great Plains. Agron J 99:1684-1699.

Fehr W and Caviness C (1977) Stages of Soybean Development. Special Report n. 80. Iowa State University, Ames, 11 pp.

Franchini JC, Debias H, Sacoman A, Nepomuceno AL and Farias JRB (2009) Manejo do Solo para Redução das Perdas de Produtividade pela Seca. Embrapa Soja, Londrina, 39 pp.

Fujita M, Fujita Y, Maruyama K, Seki M, Hiratsu K, Ohme-Takagi M, Tran L-SP, Yamaguchi-Shinozaki K and Shinozaki K (2004) A dehydration-induced NAC protein, RD26, is involved in a novel ABA-dependent stress-signaling pathway. Plant J 39:863-876.

Fujita Y, Fujita M, Satoh R, Maruyama K, Parvez MM, Seki M, Hiratsu K, Ohme-Takagi M, Shinozaki K and YamaguchiShinozaki K (2005) AREB1 is a transcription activator of novel ABRE-dependent ABA signaling that enhances drought stress tolerance in Arabidopsis. Plant Cell 17:34703488.

Guiltinan M, Marcotte W and Quatrano R (1990) A plant leucine zipper protein that recognizes an abscisic acid response element. Science 250:267-271.

Hellemans J, Mortier G, De Paepe A, Speleman F and Vandesompele J (2007) qBase relative quantification framework and software for management and automated analysis of realtime quantitative PCR data. Genome Biol 8:R19.

Higo K, Ugawa Y, Iwamoto M and Korenaga T (1999) Plant cis-acting regulatory DNA elements (PLACE) database: 1999. Nucleic Acids Res 27:297-300.

Hruz T, Laule O, Szabo G, Wessendorp F, Bleuler S, Oertle L, Widmayer P, Gruissem W and Zimmermann P (2008) Genevestigator V3: A reference expression database for the meta-analysis of transcriptomes. Adv Bioinform 2008:e420747.

Huang G-T, Ma S-L, Bai L-P, Zhang L, Ma H, Jia P, Liu J, Zhong $\mathrm{M}$ and Guo Z-F (2011) Signal transduction during cold, salt, and drought stresses in plants. Mol Biol Rep 39:969-987. 
Ito Y, Katsura K, Maruyama K, Taji T, Kobayashi M, Seki M, Shinozaki K and Yamaguchi-Shinozaki K (2006) Functional analysis of rice DREB1/CBF-type transcription factors Involved in cold-responsive gene expression in transgenic rice. Plant Cell Physiol 47:141-153.

Kang JY, Choi HI, Im MY and Kim SY (2002) Arabidopsis basic leucine zipper proteins that mediate stress-responsive abscisic acid signaling. Plant Cell 14:343-357.

Kankainen M and Holm L (2004) POBO, transcription factor binding site verification with bootstrapping. Nucleic Acids Res 32:W222-W229.

Kulcheski FR, Marcelino-Guimaraes FC, Nepomuceno AL, Abdelnoor RV and Margis R (2010) The use of microRNAs as reference genes for quantitative polymerase chain reaction in soybean. Anal Biochem 406:185-192.

Larkin MA, Blackshields G, Brown NP, Chenna R, McGettigan PA, McWilliam H, Valentin F, Wallace IM, Wilm A, Lopez $\mathrm{R}$, et al. (2007) Clustal W and clustal X ver. 2.0. Bioinformatics 23:2947-2948.

Lenka S, Lohia B, Kumar A, Chinnusamy V and Bansal K (2009) Genome-wide targeted prediction of ABA responsive genes in rice based on over-represented cis-motif in co-expressed genes. Plant Mol Biol 69:261-271.

Li YH, Lee KK, Walsh S, Smith C, Hadingham S, Sorefan K, Cawley G and Bevan MW (2006) Establishing glucose- and ABA-regulated transcription networks in Arabidopsis by microarray analysis and promoter classification using a Relevance Vector Machine. Genome Res 16:414-427.

Li Z-Y and Chen S-Y (1999) Inducible expression of translation elongation factor $1 \mathrm{~A}$ gene in rice seedlings in response to environmental stresses. Acta Bot Sin 41:800-806.

Mahajan S and Tuteja N (2005) Cold, salinity and drought stresses: An overview. Arch Biochem Biophys 444:139-158.

Manavalan LP, Guttikonda SK, Tran LSP and Nguyen HT (2009) Physiological and molecular approaches to improve drought resistance in soybean. Plant Cell Physiol 50:1260-1276.

Martins PK, Jordao BQ, Yamanaka N, Farias JRB, Beneventi MA, Binneck E, Fuganti R, Stolf R and Nepomuceno AL (2008) Differential gene expression and mitotic cell analysis of the drought tolerant soybean (Glycine max L. Merrill Fabales, Fabaceae) cultivar MG/BR46 (Conquista) under two water deficit induction systems. Genet Mol Biol 31:512-521.

Mochida K, Yoshida T, Sakurai T, Yamaguchi-Shinozaki K, Shinozaki K and Tran L-SP (2009) LegumeTFDB: An integrative database of Glycine max, Lotus japonicus and Medicago truncatula transcription factors. Bioinformatics 26:290-291.

Msanne J, Lin J, Stone J and Awada T (2011) Characterization of abiotic stress-responsive Arabidopsis thaliana RD29A and $R D 29 B$ genes and evaluation of transgenes. Planta 234:97107.

Munns R, James RA, Sirault XRR, Furbank RT and Jones HG (2010) New phenotyping methods for screening wheat and barley for beneficial responses to water deficit. J Exp Bot 61:3499-3507.

Nakashima K, Fujita Y, Katsura K, Maruyama K, Narusaka Y, Seki M, Shinozaki K and Yamaguchi-Shinozaki K (2006) Transcriptional regulation of $\mathrm{ABI} 3$-and ABA-responsive genes including RD29B and RD29A in seeds, germinating embryos, and seedlings of Arabidopsis. Plant Mol Biol 60:51-68.

Pellegrineschi A, Reynolds M, Pacheco M, Brito RM, Almeraya R, Yamaguchi-Shinozaki K and Hoisington D (2004) Stress-induced expression in wheat of the Arabidopsis thaliana DREB1A gene delays water stress symptoms under greenhouse conditions. Genome 47:493-500.

Qin F, Kakimoto M, Sakuma Y, Maruyama K, Osakabe Y, Tran L-SP, Shinozaki K and Yamaguchi-Shinozaki K (2007) Regulation and functional analysis of ZmDREB2A in response to drought and heat stresses in Zea mays L. Plant J 50:54-69.

Rodrigues FA, Marcolino J, Carvalho JdFC, Nascimento LCd, Neumaier N, Farias JRB, Carazzolle MF, Marcelino FC and Nepomuceno AL (2012) Using subtractive libraries to prospect differentially expressed genes in soybean plants submitted to water deficit. Genet Mol Biol 35(suppl 1): 304314.

Seki M, Kamei A, Yamaguchi-Shinozaki K and Shinozaki K (2003) Molecular responses to drought, salinity and frost: Common and different paths for plant protection. Curr Opin Biotechnol 14:194-199.

Shinozaki K and Yamaguchi-Shinozaki K (2007) Gene networks involved in drought stress response and tolerance. J Exp Bot 58:221-227.

Simpson SD, Nakashima K, Narusaka Y, Seki M, Shinozaki K and Yamaguchi-Shinozaki K (2003) Two different novel cis-acting elements of erd1, a clpA homologous Arabidopsis gene function in induction by dehydration stress and dark-induced senescence. Plant J 33:259-270.

Sitnikova T, Rzhetsky A and Nei M (1995) Interior-branched and bootstrap tests of phylogenetic trees. Mol Biol Evol 12:319333.

Stolf-Moreira R, Medri M, Neumaier N, Lemos N, Brogin R, Marcelino F, de Oliveira M, Farias J, Abdelnoor R and Nepomuceno A (2010a) Cloning and quantitative expression analysis of drought-induced genes in soybean. Genet Mol Res 11:858-867.

Stolf-Moreira R, Medri M, Neumaier N, Lemos N, Pimenta J, Tobita S, Brogin R, Marcelino-Guimarães F, Oliveira M, Farias J, et al. (2010b) Soybean physiology and gene expression during drought. Genet Mol Res 5:1946-1956.

Stolf-Moreira R, Lemos E, Carareto-Alves L, Marcondes J, Pereira S, Rolla A, Pereira R, Neumaier N, Binneck E, Abdelnoor $\mathrm{R}$, et al. (2011) Transcriptional profiles of roots of different soybean genotypes subjected to drought stress. Plant Mol Biol Rep 29:19-34.

Tamura K, Dudley J, Nei M and Kumar S (2007) MEGA4: Molecular Evolutionary Genetics Analysis (MEGA) software ver. 4.0. Mol Biol Evol 24:1596-1599.

Texeira LR, Braccini AdLe, Sperandio D, Scapim CA, Schuster I and Viganó J (2008) Avaliação de cultivares de soja quanto à tolerância ao estresse hídrico em substrato contendo polietileno glicol. Acta Sci Agron 30:217-223.

Tran L-S, Quach T, Guttikonda S, Aldrich D, Kumar R, Neelakandan A, Valliyodan B and Nguyen H (2009) Molecular characterization of stress-inducible GmNAC genes in soybean. Mol Genet Genomics 281:647-664.

Umezawa T, Nakashima K, Miyakawa T, Kuromori T, Tanokura M, Shinozaki K and Yamaguchi-Shinozaki K (2010) Molecular basis of the core regulatory network in ABA responses: 
Sensing, signaling and transport. Plant Cell Physiol 51:1821-1839.

Untergasser A, Nijveen H, Rao X, Bisseling T, Geurts R and Leunissen JAM (2007) Primer3Plus, an enhanced web interface to Primer3. Nucleic Acids Res 35:W71-W74.

Urano K, Maruyama K, Ogata Y, Morishita Y, Takeda M, Sakurai N, Suzuki H, Saito K, Shibata D, Kobayashi M, et al. (2009) Characterization of the ABA-regulated global responses to dehydration in Arabidopsis by metabolomics. Plant J 57:1065-1078.

Xu H, Li Y, Yan Y, Wang K, Gao Y and Hu Y (2010) Genome-scale identification of Soybean BURP domain-containing genes and their expression under stress treatments. BMC Plant Biol 10:e197.

Yamaguchi-Shinozaki K and Shinozaki K (2006) Transcriptional regulatory networks in cellular responses and tolerance to dehydration and cold stresses. Annu Rev Plant Biol 57:781803.

Zhao S and Fernald RD (2005) Comprehensive algorithm for quantitative real-time polymerase chain reaction. J Comput Biol 12:1047-1064.

\section{Internet Resources}

ClustalW2 site, http://www.ebi.ac.uk/Tools/clustalw2/index.html (May 11, 2011).

Genevestigator web tool, https:www.genevestigator.com/gv/index.jsp (July 11, 2011).

Phytozome, http://www.phytozome.net/soybean v5.0 (December $15,2010)$.
Plant Cis program-acting Regulatory DNA Elements, PLACE, http//www.dna.affrc.go.jp/PLACE (September 1, 2010).

Pobo web tool, http://ekhidna.biocenter.-helsinki.fi:9801/pobo (May 15, 2011).

The Arabidopsis Information Resource, TAIR, http://www.arabidopsis.org (December 15, 2010).

Brazilian Institute of Geography and Statistics (IBGE), http://www.ibge.gov.br (October 20, 2010).

\section{Supplementary Material}

The following online material is available for this article:

Table S1 - Cis-regulatory element responsive to drought stress, salinity stress, osmotic stress and ABA.

Table S2 - Frequency of the cis-elements present in promoters of the DMGs.

Figure S1 - Search strategy for DMGs involved in the soybean response to water deficit.

Figure S2 -Digital expression pattern of Arabidopsis DMGs involved in the response to different water privation conditions and ABA stimulus

This material is available as part of the online article from http://www.scielo.br/gmb.

License information: This is an open-access article distributed under the terms of the Creative Commons Attribution License, which permits unrestricted use, distribution, and reproduction in any medium, provided the original work is properly cited. 
Table S1 - Cis-regulatory elements responsive to drought stress, salinity stress, osmotic stress and ABA.

\begin{tabular}{|c|c|}
\hline Pattern & Description pattern \\
\hline ACGT & This sequence required for etiolation-induced expression of erd 1. \\
\hline ACCGAC & DBF2" bound to "DRE2"; rab17 is expressed during late embryogenesis, and is induced by ABA. \\
\hline WAACCA & MYB recognition site found in the promoters of the dehydration-responsive gene $\mathrm{rd} 22$. \\
\hline CANNTG & $\begin{array}{l}\text { MYC recognition site found in the promoters of the dehydration-responsive gene rd22 and many other } \\
\text { genes in Arabidopsis; Binding site of ATMYC2. }\end{array}$ \\
\hline ACGTGKC & DRE and ABRE are interdependent in the ABA-responsive expression of the rd29A. \\
\hline YAACKG & MYB recognition site found in the promoters of the dehydration-responsive gene $\mathrm{rd} 22$. \\
\hline RYACGTGGYR & ABRE in Arabidopsis dehydration-responsive gene rd22. \\
\hline CCACGTGG & ABRE; $\mathrm{ABA}$ and water-stress responses. \\
\hline YACGTGGC & ABA responsive element found is the promoter of stress regulated. \\
\hline CACATG & MYC binding site in rd22 gene of Arabidopsis thaliana; ABA-induction. \\
\hline CATGTG & MYC recognition sequence necessary for expression of erd 1 in dehydrated Arabidopsis. \\
\hline CNGTTR & ATMYB2 is involved in regulation of genes that are responsive to water stress in Arabidopsis. \\
\hline CCGAC & Core of low temperature responsive element (LTRE) of cor15a gene in Arabidopsis. \\
\hline TACCGACAT & Related to responsiveness to drought, low-temperature or high-salt stress. \\
\hline TAACTG & ATMYB2 is involved in regulation of genes that are responsive to water stress in Arabidopsis. \\
\hline CTAACCA & Binding site for MYB in dehydration-responsive gene, $\mathrm{rd} 22$. \\
\hline WK[TAGC]CGTR & NAC binding site (NACBS). \\
\hline
\end{tabular}

Note: The symbol $\mathrm{W}$ was used in addition to $\mathrm{A}$ or $\mathrm{T}$; the symbol $\mathrm{R}$ was used in addition to $\mathrm{A}$ or $\mathrm{G}$; the symbol $\mathrm{Y}$ was used in addition to $\mathrm{C}$ or $\mathrm{T}$; the symbol $\mathrm{K}$ was used in addition to $\mathrm{G}$ or $\mathrm{T}$; The symbol $\mathrm{W}$ was used in addition to $\mathrm{T}$ or $\mathrm{A}$; and the symbol $\mathrm{N}$ was used in additional to A,C,G or T. 
Table $S 2$ - Frequency of cis elements present in promoters of the DMG.

\begin{tabular}{cccccc}
\hline Motif & Data set & $\begin{array}{c}\text { Number of promoters } \\
\text { in a each dataset }\end{array}$ & $\begin{array}{c}\text { Number of promoters } \\
\text { containing the pattern }\end{array}$ & $\begin{array}{c}\text { Total number of patterns } \\
\text { in each dataset }\end{array}$ & Promoter mean \\
\hline ACGT & BG & 77222 & $55801(72.26 \%)$ & 233388 & 3.02 \\
& Cluster 1 & 3 & $3(100 \%)$ & 16 & 5.33 \\
ACCGAC & BG & 77222 & $8254(10.7 \%)$ & 10276 & 0.13 \\
& Cluster 1 & 3 & $2(66.7 \%)$ & 2 & 0.66 \\
WAACCA & BG & 77222 & $58808(76.2 \%)$ & 121330 & 1.56 \\
& Cluster 1 & 3 & $3(100 \%)$ & 6 & 2 \\
CANNTG & BG & 77222 & $73865(95.7 \%)$ & 540426 & 7.07 \\
& Cluster 1 & 3 & $3(100 \%)$ & 24 & 7.99 \\
\hline
\end{tabular}

Note: All analyzed promoter sequences presented 1,000 bp and POBO was run with the following parameters: Number pseudoclusters 50 and length of the background promoter $1.000 \mathrm{bp}$, bootstrap 1000. Calculated t-test using the linked on line GrapPad web site: ttp://www.graphpad.com/quickcalcs/DistMenu.cfm). $\mathrm{P}<0.0001$. The symbol $\mathrm{W}$ was used in addition to $\mathrm{A}$ or $\mathrm{T}$ and the symbol $\mathrm{N}$ was used in additional to $\mathrm{A}, \mathrm{C}, \mathrm{G}$ or T. BG - background set that comprise all promoters regions of the G. max genome. Cluster 1 - promoters set that comprise the promoters regions of the GOI genes. 


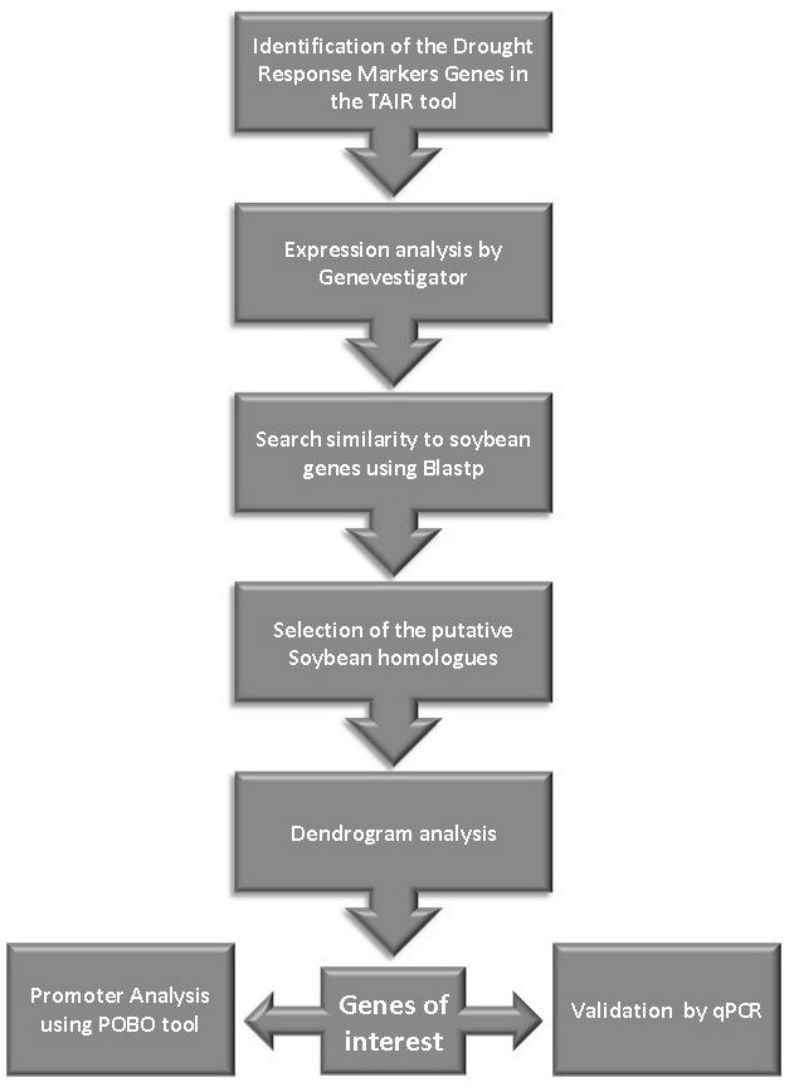

Figure S1 - Search strategy for DMGs involved in the soybean response to water deficit. 


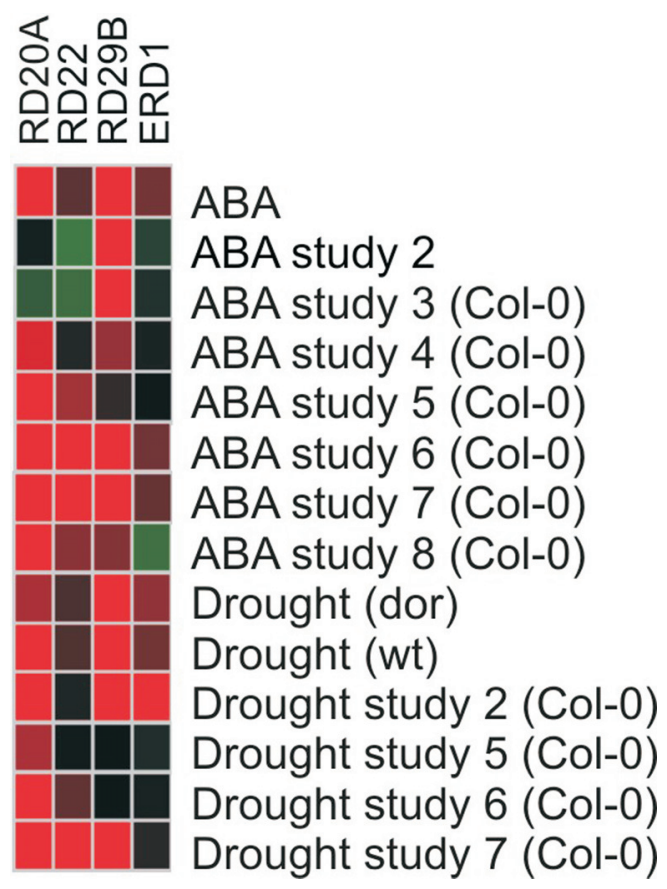

Figure S2 - Digital expression pattern of Arabidopsis DMGs involved in the response to different water privation conditions and ABA stimulus, evaluated by the Genevestigator web tool. The intensity of the red color is equivalent to increase in the gene induction in response to drought stress or ABA. The increase in the intensity of the green color is equivalent to increase in the gene repression in response to drought stress or ABA. The black color indicates that it is not induced or repressed under drought stress conditions. Description of experiments: ABA: wild type (Col-0) seedlings, treated with $10 \mu \mathrm{M}$ ABA for $1 \mathrm{~h}$; ABA study 2: Leaf samples from wild type (Col-0) plants, not watered for 7 days; ABA study 3 (Col-0): After stratification at $4{ }^{\circ} \mathrm{C}$ for 4 days, wild type seeds were allowed to germinate on the MS- $2 \%$ sucrose plate containing $0.5 \mu \mathrm{M}$ ABA for two days; ABA study 4 (Col-0): wild type leaves of 4 week-old plants, sprayed with $50 \mu \mathrm{M}$ ABA. After $4 \mathrm{~h}$, samples were collected; ABA study 5 (Col-0): Plant samples of Col-0 grown for 2 weeks on MS agar medium with 3\% sucrose and then treated with $100 \mu \mathrm{M} \mathrm{ABA}$ for $1 \mathrm{~h}$; ABA study 6 (Col-0): Plant samples of wild type (Col-0) grown for 2 weeks on MS agar medium with 3\% sucrose and then exposed to dehydration stress for $1 \mathrm{~h}$; ABA study 7 (Col-0): Isolated guard cell samples of 5-week-old wild-type plants, treated with $50 \mathrm{mM} \mathrm{ABA}$ for $3 \mathrm{~h}$; ABA study 8 (Col-0): Excised leaf samples of 5-week-old wild-type plants, treated with 50 $\mathrm{mM} \mathrm{ABA}$ for $3 \mathrm{~h}$. Drought (dor): Rosette leaf samples of dor plants, grown under normal watering conditions for 24 days and then stressed by completely depriving them of irrigation for 10 days; Drought (wt): Rosette leaf samples of wild type (Col-0) plants, grown under normal watering conditions for 24 days and then stressed by completely depriving them of irrigation for 10 days; Drought study 2 (Col-0): Leaf samples from wild-type (Col 0$)$ plants, not watered for 7 days; Drought study 5 (Col-0): Rosette leaf samples of dor plants, grown under normal watering conditions for 24 days and then stressed by completely depriving them of irrigation for 10 days; Drought study 6 (Col-0): Plant samples of Col-0 grown for 2 weeks on MS agar medium with 3\% sucrose and then exposed to dehydration stress for $1 \mathrm{~h}$; Drought study 7 (Col-0); Drought study 7 (Col-0): Plant samples of Col-0 grown for 2 weeks on MS agar medium with 3\% sucrose and then exposed to dehydration stress for $4 \mathrm{~h}$. 